\title{
Lipid kinetics in patients with non-fermenting Gram-negative bacterial ventilator-associated pneumonia
}

\author{
ILBAN Omur $^{1}$, ILBAN Aysegul² \\ Department of Intensive Care, Konya Numune Hospital, Selcuklu, Konya, Turkey. droilban@gmail.com
}

\begin{abstract}
OBJECTIVES: In non-fermenting Gram-negative bacilli (NFGNB) infections, the interaction between the immune system of the host and the bacteria plays a more important role in determining the pathogenesis than the virulence factors of bacteria per se. We aimed to evaluate the sequential changes in the inflammatory response, including the lipid profile, antimicrobial susceptibility, and outcomes in NFGNB ventilator-associated pneumonia (VAP).

METHODS: A total of 81 patients who developed NFGNB VAP were divided into two groups, namely multidrug resistant $(M D R)(n=51)$ and non-MDR $(n=30)$ groups.

RESULTS: When compared with the control group, the cholesterol levels of VAP patients decreased, while the levels of $C$-reactive protein and procalcitonin (PCT) levels significantly increased $(p<0.05)$. The best sensitivity (93\%) corresponds to PCT levels on day 4, and the best specificity (89\%) corresponds to the levels of highdensity lipoprotein (HDL) on day 4 , which makes the latter parameters suitable candidates for predicting the 30 day mortality. The impact of resistant NFGNB isolates on early and late mortality rates was similar.

CONCLUSION: In NFGNB VAP patients, the change relative to antimicrobial resistance was not appreciated in either the systemic inflammatory response or disease severity. MDR NFGNB isolates were not associated with greater mortality in VAP patients. The decrease in serum HDL and increase in PCT on day 4 indicated a poor prognosis (Tab. 5, Fig. 2, Ref. 30). Text in PDF www.elis.sk

KEY WORDS: Gram-negative bacteria, inflammatory response, lipid metabolism, multiple drug resistance, ventilator-associated pneumonia.
\end{abstract}

\begin{abstract}
Abbreviations: A. baumannii-Acinetobacter baumannii, ATS American Thoracic Society, CPIS - Clinical Pulmonary Infection Score, CRP - C-reactive protein, HDL - high-density lipoprotein, ICU - intensive care unit, LDL - low-density lipoprotein, MDR - multi-drug resistant, MV - mechanical ventilation, NFGNB non-fermenting Gram-negative bacilli, P. aeruginosa - Pseudomonas aeruginosa, $\mathrm{PCT}$ - procalcitonin, $\mathrm{VAP}$ - ventilator-associated pneumonia
\end{abstract}

\section{Introduction}

Non-fermenting Gram-negative bacilli (NFGNB) are aerobic, non-lactose fermenting, non-motile, catalase-positive, oxidasenegative coccobacilli that pose a major threat to critically ill subjects (1). NFGNBs are mainly opportunistic pathogens, which are difficult to treat due to their multiple, intrinsic or acquired drug resistance mechanisms, lack of standardization of antimicrobial

${ }^{1}$ Department of Intensive Care, Konya Numune Hospital, Selcuklu, Konya, Turkey, and ${ }^{2}$ Department of Microbiology, Selcuk University, Alaeddin Keykubad Yerleskesi, Selcuklu, Konya, Turkey

Address for correspondence: O. Ilban, MD, Department of Intensive Care, Konya Numune Hospital, Selcuklu, 42250, Konya, Turkey.

Phone: 09.0505.2227998, Fax: 09.0332.2356786 susceptibility testing for many organisms, and poor correlation between in-vitro disc diffusion test results as well as poor effectiveness of the drugs in vivo (2). Primarily, Pseudomonas aeruginosa (P. aeruginosa) and Acinetobacter baumannii (A. baumannii) are NFGNB which are potential multi-drug resistant (MDR) pathogens that are related to ventilator-associated pneumonia (VAP). Although MDR strains reduce the options of treatment and increase the risk of insufficient empirical antimicrobial treatment, the fact that early mortality is higher in infections caused by strains of $P$. aeruginosa that are non-MDR than in those caused by MDR $P$. aeruginosa, suggests that infections caused by the former may be more virulent (3-5).

Various studies have shown that different inflammatory cytokine levels may correlate to the severity of the disease and that this uncontrolled inflammatory response is an important determinant of mortality $(6,7)$. Conditions associated with the organism, host and administered treatment are determinants for the result of infection and mortality rate. The infection by resistant organisms may determine the severity of the underlying condition (3). Therefore, the interaction between the immune system of the host and the bacteria plays a more important role in determining the pathogenesis than the virulence factors of $P$. aeruginosa per se (8).

Owing to their direct immunomodulatory properties, lipoproteins and lipids bind and neutralize toxic bacterial substances (9). 
During the acute phase response and inflammation, high-density lipoprotein cholesterol (HDL) presents significant structural and functional changes (10). HDL, low-density lipoprotein cholesterol (LDL), and apo-A1 have been proven to own prognostic values in sepsis, pneumonia, and other infections (11).

There is limited literature about the inflammatory response to NFGNB infections and effects of antimicrobial resistance on the immune response and clinical outcomes. Therefore, in our study, we evaluated the relationship between the changes in serum lipid metabolism and other inflammation markers [procalcitonin (PCT) and $\mathrm{C}$-reactive protein (CRP)] with the antimicrobial resistance status and results in NFGNB VAP patients. Also, we aimed to investigate whether there is a dissimilarity in the clinical characteristics of the subjects depending on the resistance profile of NFGNB.

\section{Materials and methods}

\section{Study population}

This prospective observational cohort study was conducted on adult patients treated in intensive care unit (ICU), who were endotracheally intubated and submitted to mechanical ventilation (MV) for a minimum of 48 hours in Konya Numune Hospital ICU which has 42 beds for surgery and medicine. The guidelines of the Declaration of Helsinki were followed in the study which took place between May 2019 and December 2020 and was approved by the Ethics Committee of Necmettin Erbakan University Medical School. Also, the study was introduced in the index of the National Clinical Trial website (www.clinicaltrials.gov: NCT04748432). Informed consents were obtained from the patients who participated in the study or from their relatives.

\section{Inclusion and exclusion criteria}

ICU patients aged 18 years or older, with clinical suspicion of VAP [as defined in the American Thoracic Society (ATS) guidelines (12), and simplified version of Clinical Pulmonary Infection Score (CPIS) $>6$ (13)], with significant NFGNB growth in respiratory cultures and no symptoms or signs of infection at the time of admission were accepted in the study.

Patients suffering from chronic inflammatory disease, active tuberculosis, hepatic dysfunction, malnutrition, malignancy, severe immunosuppression (i.e., neutropenia, HIV, organ transplant, having received immunosuppressants or prednisone in a dose $\geq 20 \mathrm{mg}$ / day for two or more weeks in the preceding three months), those who within 72 hours since being included in the study had any extrapulmonary infection other than VAP, as well as those whose respiratory cultures presented bacterial agents other than NFGNB and fungal agents were not included in the study.

\section{Definitions}

Pneumonia developing after minimum 48 hours following the onset of endotracheal intubation and MV was defined as VAP (12). Among the patients with endotracheal intubation and MV for more than 48 hours, those without VAP or other nosocomial infection suspicion were defined as the control group.
Magiorakos et al were followed in the identification of MDR, extensive-drug resistant (XDR) and pan-drug resistant (PDR) pathogens (14). XDR and PDR organisms were evaluated as MDR. A detection of more than 1 NFGNB was defined as polymicrobial pneumonia. In patients with polymicrobial VAP, the finding of one or more MDR organisms was classified as MDR. The suitability of the empirical antibiotherapy was evaluated in relation to the susceptibility of the organism to antibiotics administered continuously to the patients with VAP diagnosis until obtaining the resistance results.

In our study, only the first episode of VAP was evaluated in detail. Death within 7 days from the beginning of VAP was defined as early mortality and death within 30 days for any reason, as a 30-day ICU mortality.

\section{Study protocol}

We prospectively analysed 81 consecutive patients with VAP criteria, while 50 patients with similar age and gender presenting no nosocomial infection composed the control group in the study. VAP was diagnosed clinically and confirmed microbiologically, that is, in case that the patient presented a CPIS $>6$ and the bacterial growth of tracheobronchial aspirate was $>10^{4}$ colony forming units $(\mathrm{CFU}) / \mathrm{ml}$ by the conventional culture method. The locally modified guidelines were followed for the empirical antimicrobial therapy (12).

Two consecutive samples of blood were obtained from VAP patients at the beginning of the infection and 72 hours later. Due to the possibility of antibiotic treatment altering the inflammatory markers, the samples of blood were obtained simultaneously with the diagnosis and previous to the empirical therapy. Lipid levels were analysed using Beckman Coulter AU5800 automatic biochemical analyser with the original reagents. PCT levels were analysed by Siemens ADVIA Centaur CP Immunoassay System, and CRP was measured by Siemens BN 2 plasma protein analyser system. The standard laboratory procedures of the institution were followed in the analysis of other biomarker levels. The standard microbiological methods were used in the identification of organisms isolated from tracheobronchial aspirates by quantitative cultures. In order to rule out other nosocomial infections, blood and urine were also cultured. The antimicrobial susceptibility tests were performed with VITEK 2 healthcare system (bioMérieux). The interpretation of the minimal inhibitory concentration (MIC) results was made in accordance with the breakpoints described by the European Committee on Antimicrobial Susceptibility Testing (EUCAST 2019) (15). The term antimicrobial resistance was applied to organisms with intermediate susceptibility.

\section{Sample size calculation}

For this study, the necessary sample size was determined using $\mathrm{G}^{*}$ Power statistical software, version 3.1. Before starting the study, there was no published information about the effect of antimicrobial resistance on lipid metabolism, as such, it was not possible to estimate the sample size based on previous studies. Therefore, we first conducted a pilot study on 16 patients while using the same methods as in the main study. In our preliminary 
Tab. 1. Baseline characteristics of patients with ventilator-associated pneumonia according to phenotype.

\begin{tabular}{|c|c|c|c|c|}
\hline Variables & Control & Total VAP & MDR & Non-MDR \\
\hline No & 50 & 81 & 51 & 30 \\
\hline Age in years & $57.5 \pm 14.5$ & $59.3 \pm 11.7$ & $60.1 \pm 11.5$ & $58.2 \pm 12.2$ \\
\hline Gender, male, n (\%) & $25(50)$ & $44(54)$ & $27(53)$ & $17(57)$ \\
\hline \multicolumn{5}{|l|}{ Underlying condition, $\mathrm{n}(\%)$} \\
\hline Cardiovascular & $15(30)$ & $28(35)$ & $18(35)$ & $10(33)$ \\
\hline Pulmonary & $8(16)$ & $17(21)$ & $10(20)$ & $7(23)$ \\
\hline Renal & $9(18)$ & $27(33)^{\dagger}$ & $16(31)^{\dagger}$ & $11(37)^{\dagger}$ \\
\hline Neurologic & $10(20)$ & $18(22)$ & $12(24)$ & $6(20)$ \\
\hline Diabetes mellitus & $11(22)$ & $23(28)$ & $15(29)$ & $8(27)$ \\
\hline At least one underlying condition & $35(70)$ & $63(78)$ & $41(80)$ & $22(73)$ \\
\hline Charlson index & $2.2 \pm 0.28$ & $2.3 \pm 0.37$ & $2.3 \pm 0.39$ & $2.4 \pm 0.25$ \\
\hline APACHE II & $16.3 \pm 3.2$ & $23.6 \pm 6.5^{\dagger}$ & $24 \pm 6.3^{\dagger}$ & $23.1 \pm 6.9^{\dagger}$ \\
\hline CPIS & $0.0 \pm 0.0$ & $7.3 \pm 0.28$ & $7.3 \pm 0.27$ & $7.4 \pm 0.29$ \\
\hline $\mathrm{PaO}_{2} / \mathrm{FiO}_{2}$ ratio & $282 \pm 35.7$ & $188 \pm 28.1^{\dagger}$ & $185 \pm 28^{\dagger}$ & $193 \pm 27.8^{\dagger}$ \\
\hline \multicolumn{5}{|l|}{ Main causes of ICU admission, $\mathrm{n}(\%)$} \\
\hline Medical & $37(74)$ & $63(78)$ & $39(76)$ & $24(80)$ \\
\hline Surgery/Trauma & $13(26)$ & $18(22)$ & $12(24)$ & $6(20)$ \\
\hline Positive blood culture, n (\%) & $0(0)$ & $6(7)$ & $3(6)$ & $3(10)$ \\
\hline Systemic steroids, n (\%) & $5(10)$ & $11(14)$ & $6(12)$ & $5(17)$ \\
\hline NSAID, n $(\%)$ & $5(10)$ & $6(7)$ & $4(8)$ & $2(7)$ \\
\hline Vasopressor use, n (\%) & $6(12)$ & $20(25)^{\dagger}$ & $12(24)^{\dagger}$ & $8(27)^{\dagger}$ \\
\hline Statin use, n $(\%)$ & $4(8)$ & $10(12)$ & $6(12)$ & $4(13)$ \\
\hline Microbiological persistence & $0(0)$ & $32(40)$ & $21(41)$ & $11(37)$ \\
\hline Pneumonia recurrence & $0(0)$ & $8(10)$ & $4(8)$ & $4(13)$ \\
\hline \multicolumn{5}{|l|}{ Clinical outcomes } \\
\hline Duration of intubation prior to VAP (days) & $0.0 \pm 0.0$ & $7.6 \pm 2.2$ & $8.1 \pm 2.5$ & $6.8 \pm 2.3$ \\
\hline ICU length of stay (days) & $12 \pm 5.8$ & $19.5 \pm 8.7$ & $20.1 \pm 8.7^{\dagger}$ & $18.4 \pm 8.9^{\dagger}$ \\
\hline Inadequate empirical antibiotics, n (\%) & $0(0)$ & $45(56)$ & $35(69)$ & $10(33) \div$ \\
\hline Days from VAP diagnosis to death & $0.0 \pm 0.0$ & $9.1 \pm 3.7$ & $8.9 \pm 3.6$ & $9.4 \pm 4$ \\
\hline Early mortality, n (\%) & $0(0)$ & $16(20)$ & $9(18)$ & $7(23)$ \\
\hline 30-day mortality, n (\%) & $0(0)$ & $37(46)$ & $23(45)$ & $14(47)$ \\
\hline
\end{tabular}

Tab. 2. Etiologic diagnosis of ventilator-associated pneumonia.

\begin{tabular}{lcccc}
\hline & All & MDR & Non-MDR & p value \\
\hline Non-fermenting Gram-negative bacilli & 81 & 51 & 30 & \\
\hline Polymicrobial & $15(19)$ & $10(20)$ & $5(17)$ & 0.74 \\
\hline Monomicrobial & & & & \\
$\quad$ Pseudomonas aeruginosa & $34(42)$ & $19(37)$ & $15(50)$ & 0.26 \\
Acinetobacter baumannii & $31(38)$ & $21(41)$ & $10(33)$ & 0.48 \\
Stenotrophomonas maltophilia & $2(2)$ & $1(2)$ & $1(3)$ & 1 \\
\hline
\end{tabular}

All data shown as n (\%). MDR - multi-drug resistant

study, the mean HDL levels on admission were 30.7 (SD 8.5) in the MDR group and 38.1 (SD 10.2) in the non-MDR group. In order to detect a significant difference in HDL level among the two groups, a power analysis was carried out using a 2-sided independent samples t-test with a confidence level of $95 \%(\mathrm{p}<0.05)$, effect size of $78 \%$, power of $90 \%$, and 3:5 non-MDR to MDR allocation. The sample size was finally calculated to be at least 80 patients after considering the $5 \%$ drop-out rate.

\section{Statistical analysis}

SPSS statistics package version 21.0 was used for analysing the data. Counts and percentages $(\%)$ represented categorical variables while means ( \pm standard deviation) were continuous variables. The comparison of categorical variables was made by Pearson chi-square or Fisher exact test, as suitable. Two-group comparison of normally distributed continuous variables was analysed by independent samples t-test. When the distribution was not normal, the nonparametric Mann-Whitney U test was selected. For multigroup comparisons, one way-ANOVA with Bonferroni for post hoc comparison or Kruskal-Wallis tests were used, as appropriate. The comparison of laboratory parameters evolution from day 1 to day 4 was accomplished by the paired samples t-test. Based on the results of the univariate analysis, the aptness of serum PCT and HDL levels to discern the survival status was examined by the receiver operating characteristic (ROC) curve analysis. The confidence interval (CI) of $95 \%$ and the area under the curve (AUC) were described. Youden criteria determined the adequate discriminatory cut-off values. While defining perishing as the event of interest and release from the ICU along with the ICU length of stay equal to 30 days as rightcensoring events, the Kaplan-Meier method was utilized to describe the survival of patients with NFGNB VAP (MDR vs non-MDR). The time of drawing the first positive culture from tracheobronchial aspirate culture was defined as the time of origin. A $p$ value $<0.05$ two-tailed all the significance tests.

\section{Results}

Patients developing VAP presented an average age of $59.3 \pm 11.7$ (range, 18-89 years), while $54 \%$ were male. No difference was appreciated in terms of age or sex ratio between the MDR and non-MDR groups $(\mathrm{p}<0.05)$. The baseline characteristics of patients at the onset of VAP are shown in Table 1. Fifty-one (63\%) patients had MDR NFGNB, while 30 (37\%) patients had non-MDR NFGNB. Cardiovascular disease was the most common underlying systemic disease, occurring in $35 \%$ of the patients with VAP. Compared to the control group, renal comorbidities, APACHE II scores, vasopressor usage, and ICU length of stay were significantly higher, and $\mathrm{PaO}_{2} / \mathrm{FiO}_{2}$ ratio was significantly lower in the pneumonia groups. In comparison with 
Tab. 3. Inflammatory biomarkers and antimicrobial resistance.

\begin{tabular}{|c|c|c|c|c|c|}
\hline \multirow{2}{*}{ Variables } & \multirow{2}{*}{$\begin{array}{l}\text { Control } \\
(\mathrm{n}=50)\end{array}$} & \multicolumn{2}{|c|}{$\operatorname{MDR}(n=51)$} & \multicolumn{2}{|c|}{ Non-MDR $(\mathrm{n}=30)$} \\
\hline & & Day 1 & Day 4 & Day 1 & Day 4 \\
\hline TCH (mg/dL) & $154.2 \pm 35.8$ & $124 \pm 41.7^{\dagger}$ & $129.5 \pm 33.3$ & $135 \pm 39.6^{\dagger}$ & $137 \pm 39.6$ \\
\hline $\mathrm{HDL}(\mathrm{mg} / \mathrm{dL})$ & $42 \pm 12.2$ & $32.2 \pm 8.2^{\dagger}$ & $35.2 \pm 10.8$ & $35.2 \pm 10.3^{\dagger}$ & $36.5 \pm 11.3$ \\
\hline $\mathrm{LDL}(\mathrm{mg} / \mathrm{dL})$ & $95 \pm 25.1$ & $68.2 \pm 26.8^{\dagger}$ & $77.2 \pm 27.5$ & $75 \pm 30.6^{\dagger}$ & $81.2 \pm 29.9$ \\
\hline $\mathrm{TG}(\mathrm{mg} / \mathrm{dL})$ & $131 \pm 58.8$ & $143 \pm 61.4$ & $140.9 \pm 66.2$ & $134 \pm 76$ & $136 \pm 76.1$ \\
\hline CRP (mg/L) & $44 \pm 24.8$ & $82.2 \pm 33.3^{\dagger}$ & $71.4 \pm 27.1$ & $73.2 \pm 34.7^{\dagger}$ & $59.2 \pm 28.3$ \\
\hline PCT $(\mu \mathrm{g} / \mathrm{L})$ & $0.2 \pm 0.4$ & $16 \pm 11.2^{\dagger}$ & $12.1 \pm 7.3$ & $13 \pm 9.8^{\dagger}$ & $9.3 \pm 6$ \\
\hline
\end{tabular}

Data shown as Mean \pm standard deviation. $\dagger$ control group vs pneumonia groups on day $1 ; \ddagger$ MDR vs non-MDR group on day $1, \S$ MDR vs non-MDR group on day $4(\mathrm{p}<0.05)$. Abbreviations: MDR - multi-drug resistant; TCH - total cholesterol; HDL - high-density lipoprotein cholesterol; LDL - low-density lipoprotein cholesterol; TG - triglycerides; CRP - C-reactive protein; PCT - procalcitonin

Tab. 4. Inflammatory response and outcome.

\begin{tabular}{lcccc}
\hline Variables & All & Survivors & non-survivors & p value \\
\hline No & 81 & 44 & 37 & \\
TCH on day 1 $(\mathrm{mg} / \mathrm{dL})$ & $135.1 \pm 39$ & $142.6 \pm 39.8$ & $126 \pm 36.6$ & 0.06 \\
TCH on day 4 $(\mathrm{mg} / \mathrm{dL})$ & $138.1 \pm 37.6$ & $145 \pm 44.3$ & $129.7 \pm 35.8$ & 0.07 \\
HDL on day 1 $(\mathrm{mg} / \mathrm{dL})$ & $33.6 \pm 6.8$ & $37.2 \pm 9.6$ & $29.3 \pm 6.6$ & $<0.01^{*}$ \\
HDL on day 4 $(\mathrm{mg} / \mathrm{dL})$ & $34.9 \pm 10.9$ & $41.3 \pm 11.9$ & $27.2 \pm 6.5$ & $<0.01^{*}$ \\
LDL on day 1 $(\mathrm{mg} / \mathrm{dL})$ & $69.2 \pm 24.5$ & $73.5 \pm 23.1$ & $64.4 \pm 25.5$ & 0.08 \\
LDL on day 4 $(\mathrm{mg} / \mathrm{dL})$ & $72.9 \pm 14.4$ & $75.5 \pm 19.1$ & $68.9 \pm 13.1$ & 0.09 \\
TG on day 1 $(\mathrm{mg} / \mathrm{dL})$ & $136.4 \pm 61.2$ & $130 \pm 62.3$ & $144 \pm 59.8$ & 0.31 \\
TG on day 4 $(\mathrm{mg} / \mathrm{dL})$ & $132.5 \pm 57.1$ & $127 \pm 55.5$ & $139 \pm 59.1$ & 0.36 \\
CRP on day 1 $(\mathrm{mg} / \mathrm{L})$ & $75.3 \pm 28.3$ & $70.1 \pm 19.5$ & $81.5 \pm 27.3$ & 0.09 \\
CRP on day 4 $(\mathrm{mg} / \mathrm{L})$ & $74.6 \pm 22.4$ & $70.7 \pm 21.8$ & $79.2 \pm 30.1$ & 0.11 \\
PCT on day 1 $(\mu \mathrm{g} / \mathrm{L})$ & $13.1 \pm 9.3$ & $11.1 \pm 7.8$ & $15.3 \pm 9.7$ & $0.04 *$ \\
PCT on day 4 $(\mu \mathrm{g} / \mathrm{L})$ & $12.9 \pm 9.6$ & $8.6 \pm 6.8$ & $18.2 \pm 9.5$ & $<0.01 *$ \\
\hline
\end{tabular}

Data shown as Mean \pm standard deviation. * Survivors vs non-survivors group $(\mathrm{p}<0.05)$. Abbreviations as in Table 3. the non-MDR group, APACHE II scores and ICU length of stay were higher among the MDR group; nonetheless, this dissimilitude was not statistically significant. The frequency of inadequate empirical treatment in the non-MDR group was lower than in the MDR group $(\mathrm{p}<0.01)$. No significant difference among the groups was recorded for other parameters (Tab. 1).

When the specific microbial pattern was evaluated, $19 \%$ of VAP patients were found polymicrobial. Although the number of polymicrobial agents in the MDR group was higher than in the non-MDR group (20\% vs $17 \%$, respectively), the difference was not significant $(p=0.74)$. When monomicrobial pneumonias were evaluated, although the frequency of $P$. aeruginosa was higher in the non-MDR group, that of $A$. baumannii was higher in the MDR group. Nevertheless, these differences were not found statistically significant $(\mathrm{p}=0.26, \mathrm{p}=$ 0.48 , respectively) (Tab. 2).

Serum lipid levels and inflammatory status were compared between the control and VAP groups on admission (day 1). The cholesterol levels of VAP patients decreased, while CRP and PCT levels signifi-

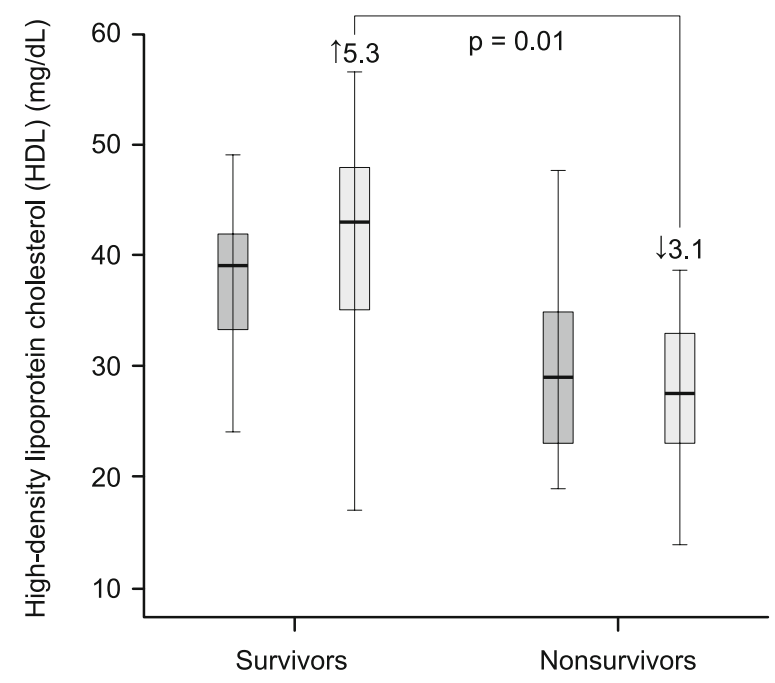

A

\section{$\square$ HDL Day $1 \quad \square$ HDL Day 4}

B

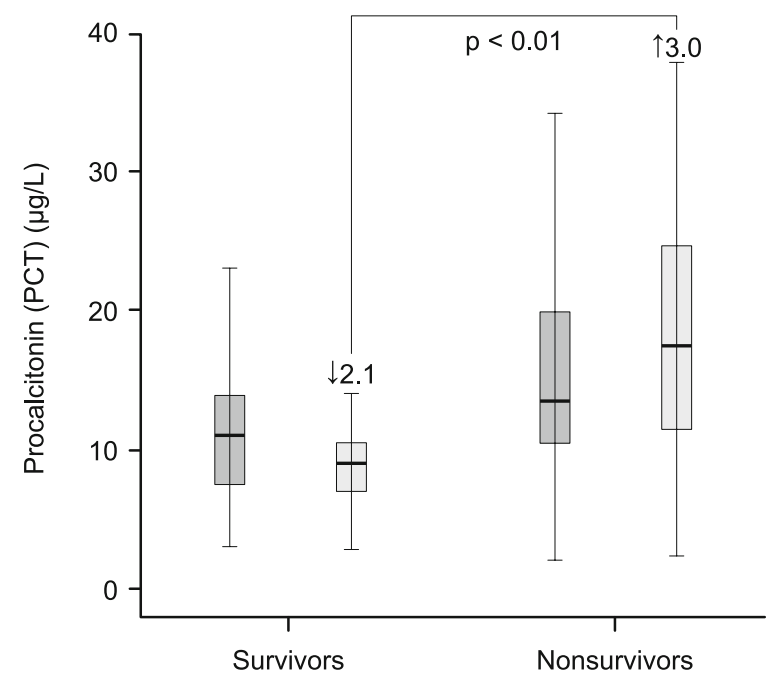

$\square$ PCT Day $1 \quad \square$ PCT Day 4

Fig. 1. Levels of serum HDL and PCT on day 1 (onset of infection) and day 4 in patients with NFGNB VAP. A. The mean change in HDL levels from day 1 to 4 in non-survivors was significantly reduced compared to survivors $(5.3 \mathrm{vs}-3.1 \mathrm{mg} / \mathrm{dL}, \mathrm{p}=0.01)$. B. The mean change in PCT levels from day 1 to day 4 in non-survivors was significant compared to survivors $(-2.1 \mathrm{vs} 3.0 \mu \mathrm{g} / \mathrm{L}, \mathrm{p}<0.01)$. NFGNB, non-fermenting Gramnegative bacilli; VAP, ventilator-associated pneumonia. 
Tab. 5. Prognostic value of statistically significant parameters.

\begin{tabular}{lccccccc}
\hline Variables & AUC & Sensitivity & Specificity & PPV & NPV & 95\% CI & p value \\
\hline HDL day 1 & 0.857 & 88 & 72 & 70 & 76 & 0.769 to 0.944 & $<0.001^{*}$ \\
HDL day 4 & 0.877 & 79 & 89 & 78 & 89 & 0.783 to 0.940 & $<0.001^{*}$ \\
PCT day 1 & 0.661 & 79 & 47 & 65 & 64 & 0.538 to 0.783 & $0.014^{*}$ \\
PCT day 4 & 0.853 & 93 & 69 & 93 & 79 & 0.755 to 0.923 & $<0.001^{*}$ \\
HDL 4+PCT 4 & 0.954 & 95 & 86 & 100 & 97 & 0.912 to 0.996 & $<0.001^{*}$ \\
\hline
\end{tabular}

Data are presented as $\%$, unless otherwise stated. $* \mathrm{p}<0.05$. Abbreviations: AUC - area under the receiver-operating-characteristic curve; $\mathrm{CI}$ - confidence interval; $\mathrm{PPV}$ - positive predictive value; NPV - negative predictive value. Abbreviations as in Table 3 .

cantly increased $(\mathrm{p}<0.05)$. However, no significant difference in the triglyceride levels was appreciated. No difference was appreciated between day- 1 and day- 4 values regarding inflammatory biomarkers in MDR and non-MDR groups (Tab. 3).

When we evaluate the relationship between inflammatory response and outcomes, HDL values were significantly higher, and PCT levels were significantly lower among survivors than among non-survivors. However, the other biomarkers presented a similar distribution in both groups (Tab. 4). The levels of HDL between days 1 and 4 decreased significantly more in non-survivors than in survivors (survivors vs non-survivors; mean change, 5.3 vs -3.1 $\mathrm{mg} / \mathrm{dL}, \mathrm{p}=0.01$ ). In addition, the PCT level variation from day 1 to day 4 was more pronounced in non-survivors than in survivors (survivors vs non-survivors; mean change, -2.1 vs $3.0 \mu \mathrm{g} / \mathrm{L}, \mathrm{p}<$ 0.01) (Fig. 1).

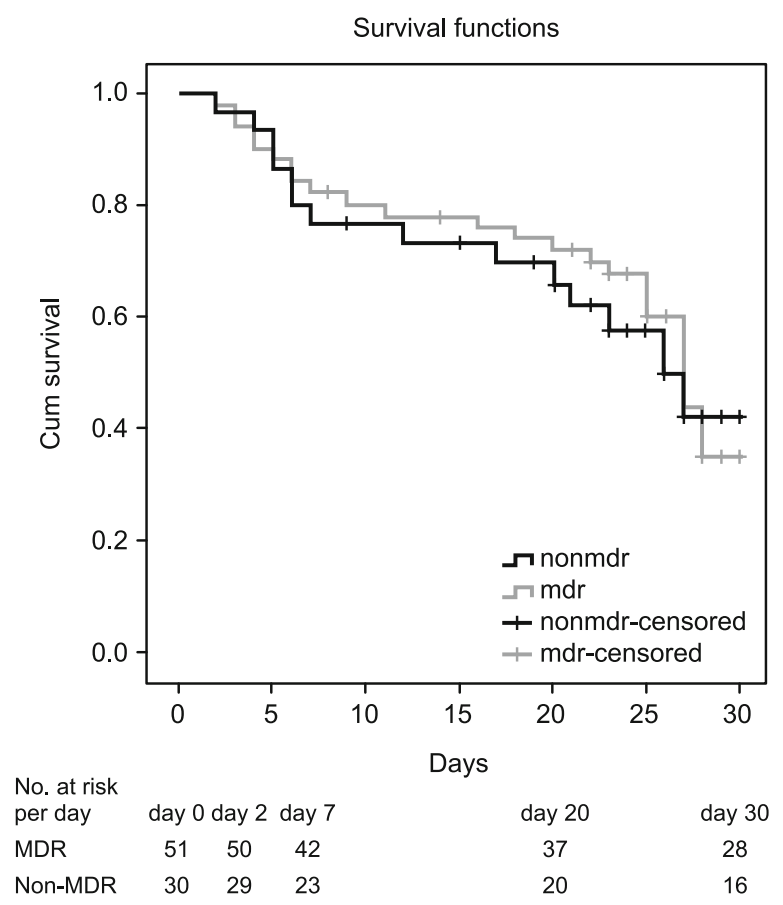

Fig. 2. The survival in ventilator-associated pneumonia patients with MDR NFGNB isolates ( $\mathrm{n}=\mathbf{5 1} ; 23$ deaths) and with non-MDRNFGNB isolates $(n=30 ; 14$ deaths) were estimated using the Kaplan-Meier method $(p=0.693$, log-rank test). MDR, multi-drug resistant; NFGNB, non-fermenting Gram-negative bacilli.
Since HDL and PCT have been shown to have a significant relationship with clinical results in VAP patients, HDL and PCT levels on days 1 and 4 were evaluated by ROC analysis as a prognostic indicator of mortality (Tab. 5). Both biomarkers reached the highest AUC values on the 4th day ( 0.877 vs 0.853 , respectively). The best sensitivity (93\%) corresponding to PCT on day 4 with a $13.5 \mu \mathrm{g} / \mathrm{L}$ cut-off value, and the best specificity ( $89 \%$ ) corresponding to HDL on day 4 with $34.5 \mathrm{mg} / \mathrm{dL}$ cut-off value could be predictors of all-cause 30-day mortality. The use of serum levels of HDL on day $4 \leq 34.5$ in combination with serum levels of PCT on day $4 \geq$ 13.5 did not improve the specificity (86\%) but resulted in $95 \%$ sensitivity with a higher AUC (0.954).

Pairwise comparison of ROC curves for predicting mortality showed no significant differences in the AUC for HDL and PCT on day 4 (the difference between areas: 0.024; SE: 0.055 ; $95 \%$ CI, $-0.085-0.132 ; z=0.426 ; p=0.67)$. Therefore, HDL and PCT could have similar prognostic values in assessing the risk of mortality in NFGNB VAP patients.

The comparison of the repercussion that antimicrobial resistance has on the mortality of patients with NFGNB pneumonia is shown in Table 1. Although mortality at early stages was more elevated in cases with non-MDR pneumonia than in the MDR group, this difference was not considered statistically significant ( $p$ $=0.54$ ). Furthermore, the overall mortality rates resembled among the groups $(\mathrm{p}=0.89)$. Time-dependent mortality analysis was also conducted. Figure 2 represents the Kaplan-Meier curves obtained for patients suffering from MDR or non-MDR VAP. The rate of early mortality was $18 \%$ for MDR strains vs $23 \%$ for non-MDR NFGNB ( $p=0.57, \log$-rank test), and the rate of 30-day ICU mortality was $45 \%$ for MDR strains vs $47 \%$ for non-MDR NFGNB (p $=0.69, \log$-rank test). Finally, the survival curve analysis confirmed that the mortality risk associated with the antimicrobial resistance pattern of the NFGNB isolate did not change.

\section{Discussion}

In this study, it was investigated whether the inflammatory biomarker levels in NFGNB infections differ according to the antimicrobial resistance status. No changes in the systemic inflammatory response and disease severity in VAP patients according to antimicrobial resistance were observed. The multi-drug resistance pattern was found to be not related to ICU mortality. We showed that the decrease in serum HDL and increase in serum PCT from day 1 to day 4 indicated a poor prognosis.

In various studies dealing with pneumonia and sepsis patients, the relationships between the structure of causative microorganisms and severity of the disease, as well as intensity of the inflammatory response, have been shown (16-18). However, the information related to the relationship between the resistance profile of NFGNB, induced inflammatory response, and clinical results is controversial. Bourboulis et al examined the relationship 
between MDR and virulence in experimental lung infection due to $P$. aeruginosa; they found that, compared to MDR isolates, in cases with susceptible isolates, the cytokine production in monocytes, apoptosis in neutrophils, pleural fluid and bacterial count in lungs and mortality increased. This was due partly to the virulence characteristics of susceptible and MDR isolates and partly to the host response (19). Zorilla et al showed that epidemic strains of MDR P. aeruginosa, defined as high-risk clones, caused a weaker inflammatory response and lower bacterial count than the susceptible strains. Nonetheless, strains with similar resistance profiles could generate different responses. Although this suggests that a fitness cost in association with MDR actually exists, the extent of the inflammatory response was not influenced by the resistance profile alone but rather with the intervention of the virulence phenotype (20).

However, in prospective studies, some differences were observed between antimicrobial resistance and inflammatory responses it induced. Zorilla et al showed that XDR P. aeruginosa infections caused a more significant increase in inflammatory markers as compared to patients with non-MDR (21). In the study of Barat et al. examining patients with ICU-acquired pneumonia, no difference in terms of either serum biomarker levels or mortality was found among MDR and non-MDR P. aeruginosa pneumonia (22). No difference according to MDR status was detected between the lipid metabolism and inflammatory biomarkers investigated in our study. These differences in the results may be related to the fact that besides the resistance fitness cost and profile, the inflammatory response is affected also by the virulence phenotype.

Studies on NFGNB infections show varying results regarding the effect of antimicrobial resistance on mortality. Zorilla et al. showed that infections caused by susceptible strains present higher mortality and that the MDR profile of $P$. aeruginosa is correlated with the fitness cost (23). Pena et al. found that the exoU type III secretion system genotype, which is the determinant of virulence of $P$. aeruginosa, is found in association with an elevated risk of early mortality, while the MDR profile is associated with late mortality (5). Tedja et al examined the effects of MDR status on the results in VAP. They demonstrated that the relation between MDR and mortality in non-fermenting Gram-negative rods was absent but MDR in other organisms was associated with increased mortality risk (24). In a recent prospective multicentre study, Dennis et al. evaluated the relationship between MDR P. aeruginosa episodes and mortality in VAP patients. Although the underlying condition in the patients was associated with mortality, there was no relationship linking the resistance profile of $P$. aeruginosa with mortality (25). Unlike other studies, Guo et al. showed that the mortality rate in patients with MDR A. baumannii bacteraemia was higher than in non-MDR patients (26). In our study, although the fact that early mortality in patients with non-MDR NFGNB pneumonia was higher than in cases with MDR pneumonias was not statistically significant, it was similar to previous studies (3, 4, 21). Furthermore, there was no relationship between the MDR status and 30-day ICU mortality. Additionally, the isolation of resistant NFGNB did not increase the risk of death as compared with cases where susceptible NFGNB was isolated.
In studies dealing with sepsis and pneumonia, HDL and PCT have been shown to be associated with mortality $(11,27)$. In our study, serum HDL had the highest AUC (0.877) and specificity $(89 \%)$ on day 4 , while serum PCT had the highest sensitivity (93\%) on day 4 . Also, the combination of HDL and PCT on day 4 for assessing the prognosis of patients with NFGNB VAP showed a higher AUC (0.954) than when assessed separately. The AUC for HDL and PCT on day 4 exhibited good discrimination between survivors and non-survivors $(>0.8)$ with a similar prognostic value $(p=0.67)$, suggesting that both biomarkers are a reliable tool in predicting the outcome in patients with NFGNB VAP.

Pena et al suggested that the effect of antimicrobial resistance may be insignificant in the presence of life-threatening problems (3). In another study, it was stated that although adequate empirical treatment was received, sensitive $P$. aeruginosa episodes had higher early mortality than MDR episodes. Even though adequate antibiotic treatment is important in VAP treatment, the severity of acute illness was shown to entail a more critical factor than the drug resistance (4). In their study on $P$. aeruginosa VAP patients, Kaminski et al found out that despite a more frequent delay in initiating adequate antimicrobial therapy, ureido/carboxypenicillin resistance was not associated with ICU or hospital mortality (28). In our study, the fact that there was no increase in ICU mortality in MDR NFGNB pneumonia despite the higher rate of inadequate empirical treatment as compared with non-MDRs, suggests that the underlying conditions may be a more important factor than the drug resistance, as stated in previous studies.

Planquette et al demonstrated that $P$. aeruginosa VAP prognosis was not affected by treatment modalities, and although $P$. aeruginosa resistance pattern did not affect $P$. aeruginosa VAP treatment insufficiency, it extended the duration of the stay in the ICU (29). In their study on ICU patients with $P$. aeruginosa pneumonia, Tumbarello et al showed that MDR was not related to ICU mortality, but it increased the MV duration and, consequently, prolonged the duration of post-infection intensive care stay (30). In our study, the ICU length of stay in MDR NFGNB pneumonia was more extended than in non-MDR, which was consistent with previous studies showing that although antimicrobial resistance did not affect mortality, it prolonged the duration of the treatment.

The current study had several strengths and some limitations. The definite diagnosis of VAP was made by quantitative cultures. The prospective and consecutive inclusion of the patients in the study limited the possible selection bias. The first limitation of our study is that the results of our single-centre study cannot be generalized to another institution or intensive care unit. Secondly, in patients with bacterial pneumonia, the fact that the accompanying viral agents are not evaluated may lead to an inflammatory response difference. In third place, factors that may affect lipid levels, such as race, familial disorders of lipid metabolism, bodymass index and nutritional status, were not evaluated. Fourthly, increased intensity and complexity of inflammatory responses seen in mechanically ventilated patients in ICU may lead to differences in the prognostic cut-off points of inflammatory markers.

In conclusion, in cases of NFGNB pneumonia, the systemic inflammatory response, including the lipid profile and disease se- 


\section{8-184}

verity, did not variate in accordance with the resistance profile. Antimicrobial resistance status in VAP patients did not adversely affect ICU mortality. HDL and PCT levels can be used as a predictor for mortality in NFGNB VAP patients.

\section{Learning points}

There was no change in systemic inflammatory response and disease severity in VAP patients according to antimicrobial resistance.

Pattern of multi-drug resistance was not associated with ICU mortality.

The decrease in serum HDL and increase in serum PCT from day 1 to day 4 indicated a poor prognosis.

\section{References}

1. Kim UJ, Kim HK, An JH, Cho SK, Park KH, Jang HC. Update on the epidemiology, treatment, and outcomes of carbapenem-resistant acinetobacter infections. Chonnam Med J 2014; 50 (2): 37-44.

2. Arora S, Gautam V, Ray P. Changing susceptibility patterns of nonfermenting gram negative bacilli. Indian J Med Microbiol 2012; 30 (4): 485-486.

3. Peña C, Suarez C, Gozalo M et al. Prospective multicenter study of the impact of carbapenem resistance on mortality in Pseudomonas aeruginosa bloodstream infections. Antimicrob Agents Chemother 2012; 56 (3): 1265-1272.

4. Peña C, Gomez-Zorrilla $S$, Oriol I et al. Impact of multidrug resistance on Pseudomonas aeruginosa ventilator-associated pneumonia outcome: predictors of early and crude mortality. Eur J Clin Microbiol Infect Dis 2013; 32 (3): 413-420.

5. Peña C, Cabot G, Gomez-Zorrilla $S$ et al. Influence of virulence genotype and resistance profile in the mortality of Pseudomonas aeruginosa bloodstream infections. Clin Infect Dis 2015; 60 (4): 539-548.

6. Remick DG, Bolgos GR, Siddiqui J, Shin J, Nemzek JA. Six at six: interleukin- 6 measured $6 \mathrm{~h}$ after the initiation of sepsis predicts mortality over 3 days. Shock 2002; 17 (6): 463-467.

7. Simon L, Gauvin F, Amre DK, Saint-Louis P, Lacroix J. Serum procalcitonin and C-reactive protein levels as markers of bacterial infection: a systemic review and meta-analysis. Clin Infect Dis 2004; 39: 206-217.

8. Rumbaugh KP, Colmer JA, Grisnold JA, Hamood AN. The effects of infection of thermal injury by Pseudomonas aeruginosa PA01 on the murine cytokine response. Cytokine 2001; 16 (4): 160-168.

9. Feingold KR, Funk JL, Moser AH, Shigenaga JK, Rapp JH, Grunfeld C. Role for circulating lipoproteins in protection from endotoxin toxicity. Infect Immun 1995; 63 (5): 2041-2046.

10. Navab M, Reddy ST, Van Lenten BJ, Anantharamaiah GM, Fogelman AM. The role of dysfunctional HDL in atherosclerosis. J Lipid Res 2009; 50 (Suppl): 145-149.

11. Morin EE, Guo L, Schwendeman A, Li XA. HDL in sepsis - risk factor and therapeutic approach. Front Pharmacol 2015; 6: 244.

12. American Thoracic Society and Infectious Diseases Society of America. Guidelines for the management of adults with hospital-acquired, ventilator associated, and healthcare-associated pneumonia. Am J Respir Crit Care Med 2005; 171 (4): 388-416.

13. Luna CM, Blanzaco D, Niederman MS et al. Resolution of ventilatorassociated pneumonia: prospective evaluation of the clinical pulmonary infection score as an early clinical predictor of outcome. Crit Care Med 2003; 31 (3): 676-682.
14. Magiorakos AP, Srinivasan A, Carey RB et al. Multidrug-resistant, extensively drug-resistant and pandrug-resistant bacteria: an international expert proposal for interim standard definitions for acquired resistance. Clin Microbiol Infect 2012; 18 (3): 268-281.

15. European Committee on Antimicrobial Susceptibility Testing. European Society of Clinical Microbiology and Infectious Diseases. Clinical Breakpoints 2019.

16. Endeman H, Meijvis SC, Rijkers GT et al. Systemic cytokine response in patients with community-acquired pneumonia. Eur Respir J 2011; 37 (6): 1431-1438.

17. Menéndez R, Sahuquillo-Arce JM, Reyes $S$ et al. Cytokine activation patterns and biomarkers are influenced by microorganisms in communityacquired pneumonia. Chest 2012; 141 (6): 1537-1545.

18. Kellum JA, Kong L, Fink MP et al. Understanding the inflammatory cytokine response in pneumonia and sepsis: results of the Genetic and Inflammatory Markers of Sepsis (GenIMS) Study. Arch Intern Med 2007; 167 (15): $1655-1663$.

19. Giamarellos-Bourboulis EJ, Tzepi I, Tsovolou I et al. Impact of multidrug resistance on experimental empyema by Pseudomonas aeruginosa. Respiration 2011; 82 (1): 46-53.

20. Gómez-Zorrilla S, Calatayud L, Juan C et al. Understanding the acute inflammatory response to Pseudomonas aeruginosa infection: differences between susceptible and multidrug-resistant strains in a mouse peritonitis model. Int J Antimicrob Agents 2017; 49 (2): 198-203.

21. Gómez-Zorrilla S, Morandeira F, Castro MJ et al. Acute Inflammatory Response of Patients with Pseudomonas aeruginosa Infections: A Prospective Study. Microb Drug Resist 2017; 23 (4): 523-530.

22. Fernández-Barat L, Ferrer M, De Rosa $\mathbf{F}$ et al. Intensive care unitacquired pneumonia due to Pseudomonas aeruginosa with and without multidrug resistance. J Infect 2017; 74 (2): 142-152.

23. Gómez-Zorrilla $\mathbf{S}$, Juan $\mathbf{C}$, Cabot $\mathbf{G}$ et al. Impact of multidrug resistance on the pathogenicity of Pseudomonas aeruginosa: in vitro and in vivo studies. Int J Antimicrob Agents 2016; 47 (5): 368-74.

24. Tedja R, Nowacki A, Fraser T et al. The impact of multidrug resistance on outcomes in ventilator-associated pneumonia. Am J Infect Control 2014; 42 (5): $542-545$.

25. Denis JB, Lehingue $S$, Pauly V et al. Multidrug-resistant Pseudomonas aeruginosa and mortality in mechanically ventilated ICU patients. Am J Infect Control 2019; 47 (9): 1059-1064.

26. Guo N, Xue W, Tang D, Ding J, Zhao B. Risk factors and outcomes of hospitalized patients with blood infections caused by multidrug-resistant Acinetobacter baumannii complex in a hospital of Northern China. Am J Infect Control 2016; 44 (4): 37-39.

27. Seligman R, Meisner M, Lisboa TC et al. Decreases in procalcitonin and C-reactive protein are strong predictors of survival in ventilator associated pneumonia. Crit Care 2006; 10 (5): 125.

28. Kaminski C, Timsit JF, Dubois Y et al. Impact of ureido/carboxypenicillin resistance on the prognosis of ventilator-associated pneumonia due to Pseudomonas aeruginosa. Crit Care 2011; 15 (2): 112.

29. Planquette B, Timsit JF, Misset BY et al. Pseudomonas aeruginosa ventilator-associated pneumonia. predictive factors of treatment failure. Am J Respir Crit Care Med 2013; 188 (1): 69-76.

30. Tumbarello M, De Pascale G, Trecarichi EM et al. Clinical outcomes of Pseudomonas aeruginosa pneumonia in intensive care unit patients. Intensive Care Med 2013; 39 (4): 682-692.

Received September 29, 2021. Accepted November 23, 2021. 